\title{
Bounds for the Lonely Runner Problems Via Linear Programming
}

\author{
Felipe Gonçalves ${ }^{1}$ • João P. G. Ramos ${ }^{2}$
}

Received: 4 January 2021 / Accepted: 17 July 2021 / Published online: 5 August 2021

(c) The Author(s) 2021

\begin{abstract}
In this note we develop a linear programming framework to produce upper and lower bounds for the lonely runner problem.
\end{abstract}

Keywords Linear programming · Lonely runner - Gap of loneliness · Dirichlet's approximation theorem . View obstruction

\section{The Lonely Runner Problem}

Suppose you are competing in race on a circular track of perimeter 1 with $n-1$ other runners. Assume all competitors have distinct constant speeds. The gap of loneliness is the largest length $\ell$ such that at some time $t$ in the future (assuming the race continues forever) the closest runner to you is at distance $\ell$. The lonely runner conjecture states that

$$
\ell \geq \frac{1}{n}
$$

This problem was introduced independently by Wills (1967) and Cusick (1973) in the context of view obstruction problems. The conjecture is known to be true for $n \leq 7$ runners. Moreover, speeds can be assumed to be distinct integers. For more on the history of partial results see Bohman et al. (2001), Perarnau and Serra (2016) and Tao (2018).

By Galilean relativity your speed can be assumed to be zero and the conjecture takes the following equivalent formulation: Let $\|x\|=\min _{n \in \mathbb{Z}}|x-n|$ denote the distance to the nearest integer. For a vector $\boldsymbol{x} \in \mathbb{R}^{n-1}$ let

$$
\mu(\boldsymbol{x})=\min \left\{\left\|x_{1}\right\|, \ldots,\left\|x_{n-1}\right\|\right\} .
$$

Felipe Gonçalves

goncalve@math.uni-bonn.de

João P. G. Ramos

joao.ramos@math.ethz.ch

1 Hausdorff Center for Mathematics, Universität Bonn, Endenicher Allee 60, Bonn 53115,

Germany

2 Department of Mathematics, ETH Zürich, Rämistrasse 101, Zürich 8092, Switzerland 
Then for any vector $\boldsymbol{v}=\left(v_{1}, \ldots, v_{n-1}\right) \in \mathbb{Z}^{n-1}$ of distinct integers show that

$$
\operatorname{gap}(\boldsymbol{v}):=\max _{t \in \mathbb{T}} \mu(t \boldsymbol{v}) \geq \frac{1}{n}, \quad(\text { conjecture })
$$

where $\mathbb{T}=\mathbb{R} / \mathbb{Z}$.

In general, since $t \mapsto \mu(t \boldsymbol{v})$ is piece-wise linear with slopes drawn from $\left\{v_{1}, \ldots, v_{n-1}\right\}$, the set of local maxima of $t \mapsto \mu(t \boldsymbol{v})$ is contained in the intersection of any two line segments. Therefore, if $t$ satisfies $\mu(t \boldsymbol{v})=\operatorname{gap}(\boldsymbol{v})$ then $t \in \frac{1}{q} \mathbb{Z}$, where $q$ is a factor of some $\left(v_{j} \pm v_{i}\right)$ with $i \neq j$, and therefore $\operatorname{gap}(\boldsymbol{v})=a / b$ where $b$ divides $q$. It is easy to show the trivial bound $\operatorname{gap}(v) \geq \frac{1}{2(n-1)}$ (see Tao (2018)[Proposition 1.1]).

\subsection{The Linear Programming Approach}

We want to study the following two problems:

Problem (Linear Programming Problems) Fix a sign ${ }^{1} \epsilon= \pm 1$. Let $\boldsymbol{v}=\left(v_{1}, \ldots, v_{n-1}\right)$ be a given vector of increasing integer speeds. We want to

$$
\text { Minimize } \epsilon \frac{\widehat{f}(0)}{f(0)}
$$

subjected to

(I) $f$ is a non-zero, even and real trigonometric polynomial

$$
f(x)=\sum_{n=-D}^{D} \widehat{f}(k) e^{2 \pi i k x}=\widehat{f}(0)+2 \sum_{k=1}^{D} \widehat{f}(k) \cos (2 \pi k x)
$$

of degree at most $D$.

(II) In case $\epsilon=+1$ we ask

$$
f(x) \geq 0 \text { for } 0 \leq x \leq \frac{1}{2}
$$

and in case $\epsilon=-1$ we ask

$$
f(x) \leq 0 \text { for } \frac{1}{v_{n-1}+v_{n-2}} \leq x \leq \frac{1}{2}
$$

(III) $\epsilon \widehat{f}(k) \leq 0$ if $k \notin\left\{0, v_{1}, \ldots, v_{n-1}\right\}$.

We denote by $\Lambda_{\epsilon}(v)$ the class of trigonometric polynomials satisfying (I), (II) and (III). We write

$$
\lambda_{+}(\boldsymbol{v})=\inf _{f \in \Lambda_{+}(\boldsymbol{v})} \frac{\widehat{f}(0)}{f(0)} \text { and } \lambda_{-}(\boldsymbol{v})=\sup _{f \in \Lambda_{-}(\boldsymbol{v})} \frac{\widehat{f}(0)}{f(0)}
$$

\footnotetext{
1 We will often identify the integers \pm 1 with the signs \pm .
} 
Theorem 1 Let $\boldsymbol{v}=\left(v_{1}, \ldots, v_{n-1}\right)$ be a vector of increasing positive integers. Then

$$
\operatorname{gap}(\boldsymbol{v}) \leq \lambda_{+}(\boldsymbol{v})
$$

and

$$
\operatorname{gap}(\boldsymbol{v}) \geq \lambda_{-}(\boldsymbol{v}) .
$$

Theorem 2 Let $\boldsymbol{v}=\left(v_{1}, \ldots, v_{n-1}\right)$ be a vector of increasing positive integers. Then equality is attained in (1) if one of the following conditions hold:

(i) All $v_{i}$ 's are odd. In this case $f(x)=\cos \left(\pi v_{i} x\right)^{2}$ is optimal for any $i=1, \ldots, n-$ 1 ;

(ii) There exist coprime integers $a, m \geq 1$ such that $a\{1, \ldots, m-1\} \subset\left\{v_{1}, \ldots, v_{n-1}\right\}$ and all integers in $\left\{v_{1}, \ldots, v_{n-1}\right\} \backslash a\{1, \ldots, m-1\}$ are not divisible by $m$. In this case $f(x)=K_{m}(a x)$ is optimal, where $K_{m}$ is Fejer's kernel (3).

(iii) There exists integer $a \geq 1$ such that $\boldsymbol{v}=a \boldsymbol{v}^{\prime}$ and $\boldsymbol{v}^{\prime}$ satisfies condition (i) or (ii). In this case if $f(x)$ is optimal for $\boldsymbol{v}^{\prime}$ then $f(a x)$ is optimal for $\boldsymbol{v}$.

In the range $n \leq 20$ and $\max \left(v_{1}, \ldots, v_{n}\right) \leq 40$ we have performed a computer search in $\boldsymbol{v}$ in conjunction with Gurobi's linear programming solver Gurobi (2020) to approximate $\lambda_{+}(\boldsymbol{v})$. The sign conditions of $f$ was modelled with sampling. This produced reliable numerical approximations to what we believe is the true value of $\lambda_{+}(\boldsymbol{v})$. In this way we check that the only cases where

$$
\operatorname{gap}(\boldsymbol{v})+[\text { very small error }]>\lambda_{+}(\boldsymbol{v})
$$

for $|\boldsymbol{v}|_{\infty} \leq 40$ and $n \leq 20$ were the ones contemplated by Theorem 2 . This leads to the following conjecture.

Conjecture Equality is attained in (1) if and only if one of the conditions in Theorem 2 hold.

It is unfortunate that the bounds generated for $\lambda_{-}(\boldsymbol{v})$ do not seem to be nearly as good as the bounds generated by $\lambda_{+}(\boldsymbol{v})$, and we believe this is because condition (II) seems to be very strong for $\epsilon=-1$. We need high degree polynomials and a large number of sampling points for feasibility of the linear program. In Sect. 3 we propose an improved version of this lower bound.

\section{Proofs for the Main Results}

We start by recalling that Dirichlet's Approximation Theorem implies the Lonely Runner Conjecture is sharp; a rephrasing of Dirichlet's theorem is

$$
\max _{t \in \mathbb{T}} \mu(t(1,2, \ldots, n-1))=\frac{1}{n} .
$$


The maxima is attained for $t=a / n$ for $a$ coprime with $n$. We now observe that inequality (1) is also tight in case $v=(1,2, \ldots, n-1)$, and Fejér's kernel

$$
K_{n}(x)=\frac{1}{n}\left(\frac{\sin (\pi n x)}{\sin (\pi x)}\right)^{2}=\sum_{|j|<n-1}(1-|j| / n) e^{2 \pi i j x}
$$

is the unique optimum. Optimality can easily be checked by hand, while uniqueness (modulo scaling) comes from the proof of Theorem 1 . Essentially, because $f \geq 0$, we must have $f(k / n)=f^{\prime}(k / n)=0$ for $k=1, \ldots, n$ and Fejér's kernel is the only even trigonometric polynomial of degree $n-1$ with these properties.

Proof of Theorem 1 Let $\delta=\operatorname{gap}(\boldsymbol{v})=\mu(t \boldsymbol{v})$ and $h(x)=(\delta-\|x\|)_{+}$be a 1-periodic hat function. Since $\widehat{h}(n)=(\sin (\pi \delta n) /(\pi n))^{2}$ for all $n \in \mathbb{Z}$, we have

$$
h(x)=\sum_{n \in \mathbb{Z}}\left(\frac{\sin (\pi \delta n)}{\pi n}\right)^{2} e^{2 \pi i n x} .
$$

If $f \in \Lambda_{+}(v)$ we obtain

$$
\begin{aligned}
\delta \widehat{f}(0) & =\delta \widehat{f}(0)+2 \sum_{j=1}^{n-1} \widehat{f}\left(v_{j}\right) h\left(t v_{j}\right) \geq \sum_{k=-D}^{D} \widehat{f}(k) h(t k) \\
& =\sum_{k \in \mathbb{Z}}\left(\frac{\sin (\pi \delta k)}{\pi k}\right)^{2} f(k t) \geq \delta^{2} f(0)
\end{aligned}
$$

which proves the upper bound. For the lower bound $(\epsilon=-1)$, first recall that $t=p / q$, $\delta=a / b$ and $b$ divides $q$, while $q$ is a factor of some $v_{j} \pm v_{i}$ with $j>i$ (both fractions in lowest terms). In particular $q \leq v_{n-1}+v_{n-2}$. If $f \in \Lambda_{-}(\boldsymbol{v})$ we obtain

$$
\begin{aligned}
\delta \widehat{f}(0)=\delta \widehat{f}(0)+2 \sum_{j=1}^{n-1} \widehat{f}\left(v_{j}\right) h\left(t v_{j}\right) & \leq \sum_{k=-D}^{D} \widehat{f}(k) h(t k) \\
& =\delta^{2} f(0)+\sum_{k \in \mathbb{Z} \backslash b \mathbb{Z}}\left(\frac{\sin \left(\pi \frac{a}{b} k\right)}{\pi k}\right)^{2} f\left(k \frac{p}{q}\right)
\end{aligned}
$$

However, since $\left\{k p / q \bmod 1: k \in \mathbb{Z}_{+} \backslash b \mathbb{Z}\right\}=\frac{1}{q}(\{1, \ldots, q-1\} \backslash b \mathbb{Z}), q \leq v_{n-1}+$ $v_{n-2}$ and $f(x) \leq 0$ for $1 /\left(v_{n-1}+v_{n-2}\right) \leq x \leq 1 / 2$, then $f(k p / q) \leq 0$ for $k \in \mathbb{Z} \backslash b \mathbb{Z}$. This concludes the proof.

This proof is inspired by the analytic proof of Dirichlet's approximation theorem due to Montgomery (1994). We observe that equality is attained in (1) or (2) if and only if there is $f \in \Lambda_{S}(v)$ such that:

(a) $\widehat{f}(k)=0$ if $k \notin\left\{v_{1}, \ldots, v_{n-1}\right\}$; 
(b) For some $t=p / q$ that is a global maxima of $t \mapsto \mu(t \boldsymbol{v})$, where $\operatorname{gap}(\boldsymbol{v})=a / b$ and $b$ divides $q$ (both fractions in lowest terms) we have $f(k p / q)=0$ if $k \in$ $\{1, \ldots, q-1\} \backslash b \mathbb{Z}$.

Proof of Theorem 2 Condition (i) is easy to check because when all $v_{i}$ 's are odd we have $\operatorname{gap}(\boldsymbol{v})=1 / 2$ while $\widehat{f}(0)=1 / 2$ and $f(0)=1$ for $f(x)=\cos \left(\pi v_{i} x\right)^{2}$. Next, if condition (ii) holds, then letting $\boldsymbol{u}=(1, \ldots, m-1)$ and $\boldsymbol{w}=\{\boldsymbol{v}\} \backslash\{a \boldsymbol{u}\}$ (abusing notation) we obtain

$$
\mu(t \boldsymbol{v})=\min (\mu(\operatorname{ta} \boldsymbol{u}), \mu(t \boldsymbol{w})) \leq \mu(\operatorname{ta} \boldsymbol{u}) \leq \frac{1}{m}
$$

On the other hand, since $a$ is coprime with $m$ we have $\mu\left(\frac{1}{m} a \boldsymbol{u}\right)=\frac{1}{m}$, and since $\frac{1}{m} \boldsymbol{w}$ has no integer coordinate we have $\mu\left(\frac{1}{m} \boldsymbol{w}\right) \geq \frac{1}{m}$. Therefore $\mu\left(\frac{1}{m} \boldsymbol{v}\right)=\frac{1}{m}$ and we obtain that $\operatorname{gap}(\boldsymbol{v})=\frac{1}{m}$. Now it is easy to check that $f(x)=K_{m}(\operatorname{ax})$ belongs to $\Lambda_{+}(\boldsymbol{v})$ and is optimal. Condition (iii) is trivial.

\section{Improved Lower Bounds}

Let $V_{q}$ be the class of vectors $v$ of increasing positive integers such that the global maxima of $t \mapsto \mu(t \boldsymbol{v})$ is attained at some point $t=p / q \in\left(0, \frac{1}{2}\right)$ (in lowest terms). By the proof of Theorem 1, we see that if we let $\Lambda_{-}(\boldsymbol{v}, q)$ be the class of functions satisfying the above conditions (I), (III) and

$$
\text { (II') } f(x) \leq 0 \text { for } \frac{1}{q} \leq x \leq \frac{1}{2} \text {, }
$$

then

$$
\lambda_{-}(\boldsymbol{v}, q):=\sup _{f \in \Lambda_{-}(\boldsymbol{v}, q)} \frac{\widehat{f}(0)}{f(0)} \leq \operatorname{gap}(\boldsymbol{v})
$$

for any $\boldsymbol{v} \in V_{q}$. For instance $(1, \ldots, n-1) \in V_{n}$, but some other examples of vectors in $V_{n}$ can be extracted from Goddyn and Wong (2006). They present conditions for $v$ to be tight, that is, $\operatorname{gap}(\boldsymbol{v})=\frac{1}{n}$. Some of these tight vectors characterized in Goddyn and Wong (2006)[Theorem 2.3] belong to $V_{n}$, for instance:

$$
(1,2, \ldots, n-3, n-1,2 n-4) \in V_{n} \text { if } n=2(\bmod 2 \cdot 3)
$$

$$
(1,2, \ldots, n-4, n-2, n-1,2 n-6) \in V_{n} \text { if } n=3(\bmod 2 \cdot 3 \cdot 5) .
$$

Theorem 3 Let $\boldsymbol{v}=\left(v_{1}, \ldots, v_{n-1}\right) \in V_{q}$. Then equality is attained in (4) if one of the following conditions hold:

(i) There exist integer $a \geq 1$ coprime with $q$ such that $a\{1, \ldots, q-1\} \subset\left\{v_{1}, \ldots, v_{n-1}\right\}$ and all integers in $\left\{v_{1}, \ldots, v_{n-1}\right\} \backslash a\{1, \ldots, q-1\}$ are not divisible by $q$. In this case

$$
f(x)=K_{q}(a x) \frac{1-\cos (\pi / q)^{2}}{\left(\cos (\pi a x)^{2}-\cos (\pi / q)^{2}\right)}
$$




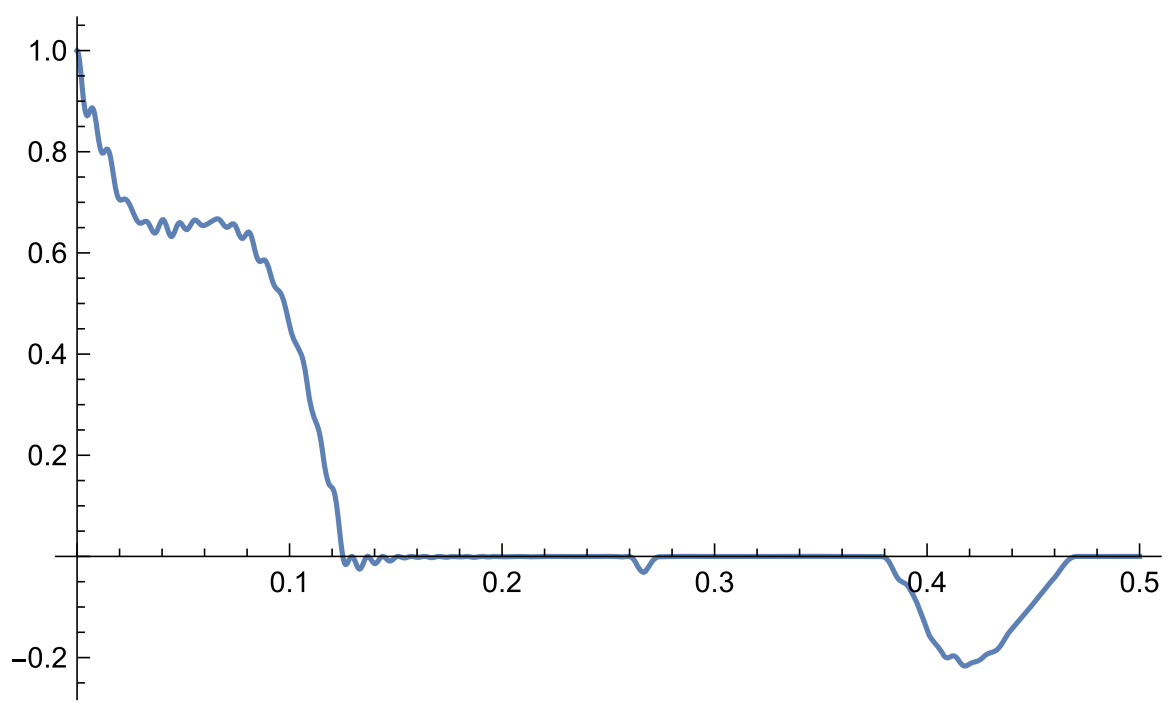

Fig. 1 This is an example of a near optimal $f$ that shows $0.125 \leq \lambda_{-}\left(1 / 8, v_{8}\right)<0.1254$ with $v_{8}=$ $(1,2,3,4,5,7,12)$

is optimal, where $K_{q}$ is Fejer's kernel (3).

(ii) There exists an integer $a \geq 1$ such that $\boldsymbol{v}=a \boldsymbol{v}^{\prime}$ and $\boldsymbol{v}^{\prime}$ satisfies condition (i). In this case if $f(x)$ is optimal for $\boldsymbol{v}^{\prime}$ then $f(a x)$ is optimal for $\boldsymbol{v}$.

Proof Assume condition (i). By the same discussion in the proof of Theorem 2 we have $\operatorname{gap}(\boldsymbol{v})=1 / q$. It is easy to see that $f$ satisfies conditions (I), (II'), (III). Moreover its mass equals the mass of $f(x / a)$, which in turn, by exact Gaussian quadrature, equals the mass of $K_{q}(x)$, which is 1 . Also $f(0)=q$. This shows optimality. Condition (ii) is trivial.

It seems that the above theorem may not be necessary for equality in (4). Indeed, if we consider the family of vectors $\boldsymbol{v}_{n}=(1,2, \ldots, n-3, n-1,2 n-4)$ then it seems that $\lambda_{-}\left(1 / n, \boldsymbol{v}_{n}\right)=\operatorname{gap}\left(\boldsymbol{v}_{n}\right)=1 / n$ for all $n=2 \bmod 6$. As an example, we get $0.125 \leq \lambda_{-}\left(1 / 8, \boldsymbol{v}_{8}\right)<0.1254$, where this is attained by the near optimal $f$ plotted in Fig. 1.

\section{Numerics}

We note that since all computations were done with high precision floating point arithmetic, we cannot know apriori how many digits of precision we have in estimating $\lambda_{ \pm}(\boldsymbol{v})$ and $\lambda_{-}(q, \boldsymbol{v})$. One reason is that we have modelled the nonnegativtivity conditions of $\epsilon f$ with sampling points (that is, we impose the constraint only in a sequence of points with very small separation) and thus, the numerical $f$ only satisfies, for instance in the $\epsilon=+1$ problem, $f \geq-\delta$ for $0 \leq x \leq \frac{1}{2}$, where $\delta>0$ is very small but easy to estimate effectively. However, transforming such a numerical estimate into an effective bound is simple in this case, all we have to do is to use instead $f_{\star}=f+\delta$, 


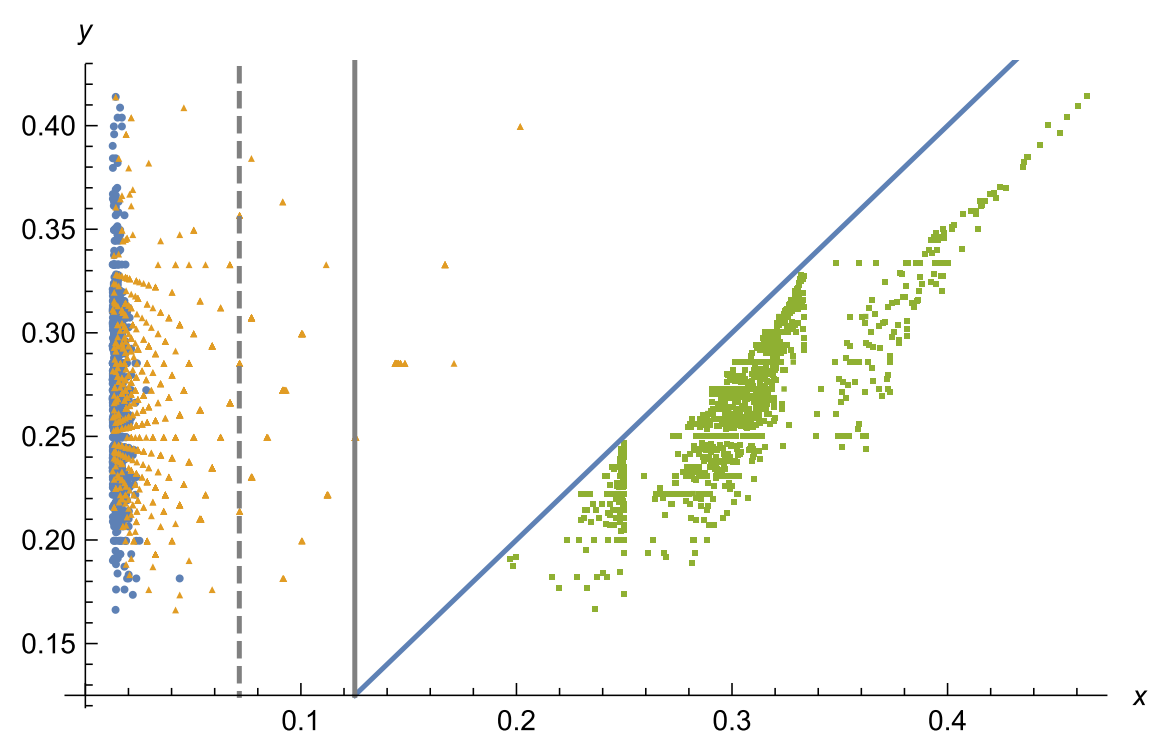

Fig. 2 The content of this scatter plot is explained in Sect. 4

as this is admissible for $\Lambda_{+}(\boldsymbol{v})$ and $\widehat{f}_{\star}(0) / f_{\star}(0)=\widehat{f}(0) / f(0)+O(\delta)$. This way we can transform our numerical estimates into precise mathematical bounds with effective error terms.

We used Gurobi (2020) to compute $\lambda_{ \pm}(\boldsymbol{v})$ and $\lambda_{-}(q, \boldsymbol{v})$ for 1000 different velocity vectors $\boldsymbol{v}=\left(v_{1}, \ldots, v_{7}\right)$ selected randomly from $0<v_{1}<\cdots<v_{7} \leq 40$ (note this is the case $n=8$, where the conjecture is not known to be true). In Fig. 2 we plot points $(x, y)$ which are numerical approximations to $\left(\lambda_{-}(\boldsymbol{v}), \operatorname{gap}(\boldsymbol{v})\right)$ in blue dots, $\left(\lambda_{-}(\boldsymbol{v}, q), \operatorname{gap}(\boldsymbol{v})\right)$ in yellow triangles and $\left(\lambda_{+}(\boldsymbol{v}), \operatorname{gap}(\boldsymbol{v})\right)$ in green squares. We took $q=$ denominator $\left(t_{\max }\right)$ where $\mu\left(t_{\max } \boldsymbol{v}\right)=\operatorname{gap}(\boldsymbol{v})$, and $q$ is the smallest with these properties. The diagonal blue line is $x=y$, the gray solid vertical line is $x=1 / 8$ and the gray dashed line if $x=1 / 14$ (the trivial lower bound). We note that we get much better lower bounds if we know a priori that $v \in V_{q}$, as the yellow triangles are clearly much closer to the line $x=y$ than the blue dots. We also see a bunch of triangles above the trivial bound and some actually proving the conjecture. The plot appears to have some interesting emergent structures: rays of triangles and parabolalike green structures. Moreover, we did not find any case where $\lambda_{+}(v)<\frac{1}{n}$, which would disprove the conjecture, and we have tested this possibility for 5000 random vectors $\boldsymbol{v}=\left(v_{1}, \ldots, v_{n-1}\right)$ with $8 \leq n \leq 10$ and $0<v_{1}<\cdots<v_{n-1}<40$ (the scatter plot of $\left(\lambda_{+}(\boldsymbol{v}), \operatorname{gap}(\boldsymbol{v})\right)$ is very similar to the green points in Fig. 2). It seems that the upper bounds are much stronger than the lower bounds and that lower bounds are only good when we know apriori (for some arithmetic reasoning) the denominator of the arg-maxima of $t \mapsto \mu(t \boldsymbol{v})$. As an example we consider $\boldsymbol{v}=(3,6,8,10,18,25,38)$ where $\operatorname{gap}(\boldsymbol{v})=\frac{2}{7}=0.285 \cdots$ is realized at $t_{\max }=\frac{3}{7}=0.428 \cdots$. The optimal functions for $\lambda_{+}(\boldsymbol{v})$ (in blue), $\lambda_{-}(1 / 7, \boldsymbol{v})$ (in yellow) and $\lambda_{-}(\boldsymbol{v})$ (in green) are presented in Fig. 3. 


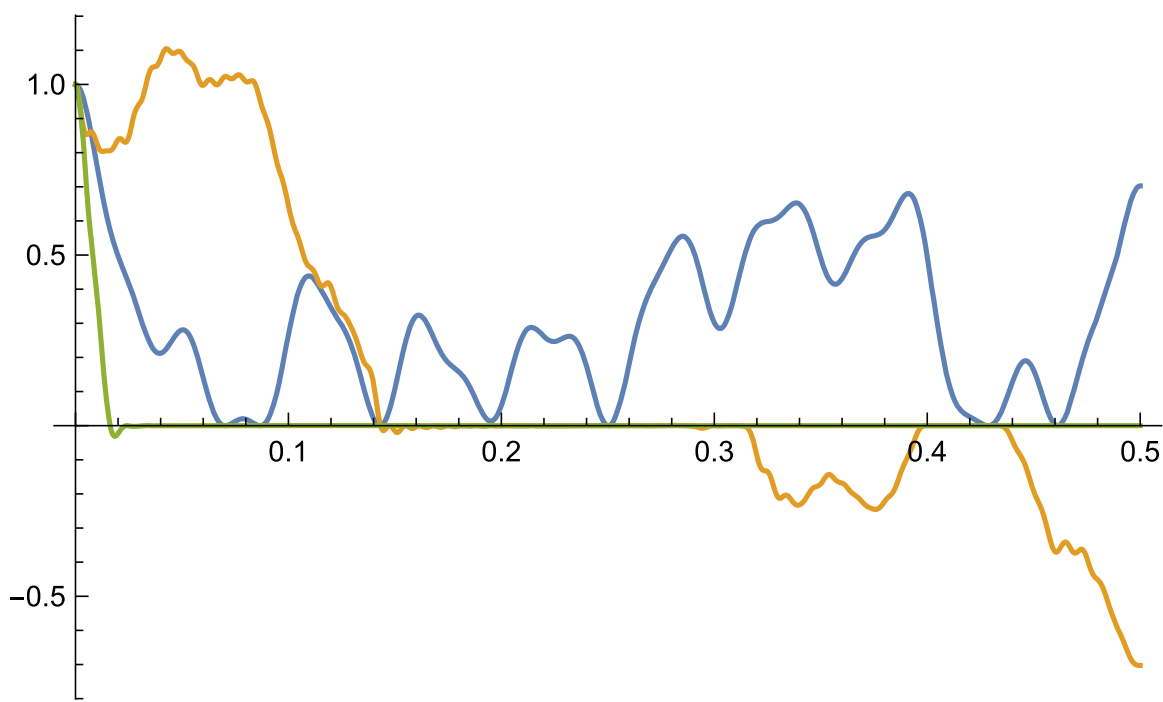

Fig. 3 As an example we consider $\boldsymbol{v}=(3,6,8,10,18,25,38)$ where $\operatorname{gap}(\boldsymbol{v})=\frac{2}{7}=0.285 \cdots$ is realized at $t_{\max }=\frac{3}{7}=0.428 \cdots$. The optimal functions for $\lambda_{+}(\boldsymbol{v})$ (in blue), $\lambda_{-}(1 / 7, \boldsymbol{v})$ (in yellow) and $\lambda_{-}(\boldsymbol{v})$ (in green) are plotted above

Acknowledgements The first author is thankful to Jeffrey Vaaler for stimulating conversations. F.G. acknowledges support from the Deutsche Forschungsgemeinschaft through the Collaborative Research Center 1060 .

Funding Open Access funding enabled and organized by Projekt DEAL.

Open Access This article is licensed under a Creative Commons Attribution 4.0 International License, which permits use, sharing, adaptation, distribution and reproduction in any medium or format, as long as you give appropriate credit to the original author(s) and the source, provide a link to the Creative Commons licence, and indicate if changes were made. The images or other third party material in this article are included in the article's Creative Commons licence, unless indicated otherwise in a credit line to the material. If material is not included in the article's Creative Commons licence and your intended use is not permitted by statutory regulation or exceeds the permitted use, you will need to obtain permission directly from the copyright holder. To view a copy of this licence, visit http://creativecommons.org/licenses/by/4.0/.

\section{References}

Bohman, T., Holzman, R., Kleitman, D.: Six lonely runners, In honor of Aviezri Fraenkel on the occasion of his 70th birthday. Electron. J. Combin. 8(2), 49 (2001)

Cusick, T.W.: View obstruction problems. Aequat. Math. 9, 165-170 (1973)

Goddyn, L., Wong, E.B.: Tight instances of the lonely runner. Integers 6, A38 (2006)

Gurobi Optimization, LLC, Gurobi Optimizer Reference Manual (2020)

Montgomery, H.L.: Ten lectures on the interface between analytic number theory and harmonic analysis. CBMS Regional Conference Series in Mathematics 84 (1994)

Perarnau, G., Serra, O.: Correlation among runners and some results on the lonely runner conjecture. Electron. J. Combin. 23(1), 22 (2016) 
Tao, T.: Some remarks on the lonely runner conjecture. Contrib. Discrete Math. 13(2), 1-31 (2018)

Wills, J.M.: Zwei Sätze über inhomogene diophantische approximation von Irrationalzahlen. Monatsch. Math. 61, 263-269 (1967)

Publisher's Note Springer Nature remains neutral with regard to jurisdictional claims in published maps and institutional affiliations. 